\title{
Case Report Peritonitis due to Neosartorya pseudofischeri in an
elderly patient undergoing peritoneal dialysis
successfully treated with voriconazole
}

\author{
B. Ghebremedhin, ${ }^{1}$ A. Bluemel, ${ }^{2}$ K.-H. Neumann, ${ }^{2}$ B. Koenig ${ }^{1}$ \\ and W. Koenig ${ }^{1}$ \\ ${ }^{1}$ Clinical Microbiology, University Clinic, Magdeburg, Germany \\ ${ }^{2}$ Division of Nephrology, University Clinic, Magdeburg, Germany
}

Correspondence

B. Ghebremedhin

beniam.ghebremedhin

@med.ovgu.de

\begin{abstract}
Aspergillus peritonitis is a rare life-threatening complication of peritoneal dialysis (PD). We report a case of symptomatic Neosartorya pseudofischeri peritonitis in a 60-year-old woman treated by continuous ambulatory peritoneal dialysis (CAPD) for 13 months, who performed peritoneal exchanges independently. This is believed to be the first published case of $N$. pseudofischeri in an elderly patient. Comprehensive treatment included early removal of the PD catheter and the use of voriconazole (200 mg Vfend twice daily) for a period of 5 weeks. This case supports the need for more effective prophylaxis and treatment of non-Candida fungal infections in CAPD patients. Our conclusions from this case and a review of the literature are that infection with this fungus can cause substantial morbidity and is best treated with prompt catheter removal, aggressive antifungal therapy with voriconazole or amphotericin $\mathrm{B}$, and vigilant observation for complications. Our report describes for what is believed to be the first time the administration of voriconazole to treat a Neosartorya peritonitis case.
\end{abstract}

Received 11 August 2008

Accepted 16 January 2009

\section{Introduction}

Peritonitis due to both intermittent and continuous ambulatory peritoneal dialysis (CAPD) remains the most common and serious complication of peritoneal dialysis (PD) (Piraino, 1998; Bibashi et al., 2003). Gram-positive bacteria are isolated in $55-80 \%$ of cases (Gloor et al., 2003; Iqbal et al., 2008), and fungal isolates in up to $10 \%$ of cases (Pimentel et al., 2005; Ram et al., 2008). In fungal peritonitis (FP), Aspergillus is rare and associated with a high mortality rate (De Hoog et al., 2000; Donnelly, 2002; Ram et al., 2008). FP is a potentially life-threatening complication of both intermittent and CAPD. Significant numbers of PD-associated cases of FP due to yeasts have been reported by several investigators (Chan et al., 1994; Garcia-Martos et al., 1991; Melez et al., 1995). Candida species account for more than $85 \%$ of the cases, whereas filamentous FP is less frequent (Bibashi et al., 2003). Although filamentous fungi causing peritonitis are reported less frequently, they encompass a wide range of agents, from the classic, systemic fungi (Bibashi et al., 1993) to the zygomycetous species (Polo et al., 1989).

The genus Neosartorya (family Trichocomaceae) was established by Malloch \& Cain (1972) to accommodate

Abbreviations: CAPD, continuous ambulatory peritoneal dialysis; CT, computed tomography; FP, fungal peritonitis; PD, peritoneal dialysis; WCC, white cell count. teleomorphs of species belonging to the section Fumigati. This section now includes the anamorphs of 11 sexual Neosartorya species and 5 asexual Aspergilli (Varga et al., 2000; Hong et al., 2008). The species are thermophilic and closely related to Aspergillus fumigatus. Neosartorya pseudofischeri has whitish, fast-growing colonies at $37^{\circ} \mathrm{C}$ and $45^{\circ} \mathrm{C}$, but not at $50{ }^{\circ} \mathrm{C}$, whereas A. fumigatus grows at all these temperatures. It is very likely that infections by Neosartorya species are underdiagnosed in clinical practice because white aspergilli are often regarded as contaminants in the laboratory (Guarro et al., 2002). The morphology of such isolates should be carefully examined by using conventional cultures on malt extract or Czapek Dox agar (Difco), and sequence-based analysis should be used for species level identification.

\section{Case report}

A 60-year-old woman was on CAPD for end-stage renal insufficiency/failure due to renovascular disease and interstitial nephritis. Her past medical history included hypertension, hyperparathyroidism, and chronic obstructive pulmonary disease. She had commenced CAPD 13 months prior to this reported episode and performed peritoneal exchanges independently. During that period, there had been multiple episodes of suspected peritonitis, each treated empirically with $1 \mathrm{~g}$ cefazolin and $40 \mathrm{mg}$ 
gentamicin, and with a single intraperitoneal dose of $500 \mathrm{mg}$ ciprofloxacin. In September 2005, she had a 3 day history of abdominal pain, accompanied by fever and nausea, and cloudy PD bags. On examination, she was found to be febrile $\left(37.8{ }^{\circ} \mathrm{C}\right)$ with a tense abdomen, rebound tenderness and decreased bowel sounds.

Initially the Tenckhoff catheter site was clean with no sign of inflammation. Full blood examination showed an elevated white cell count (WCC) of $13.8 \times 10^{6}{\text { cells } 1^{-1}}^{-1}$ and a red cell count of $4.1 \times 10^{6}$ red blood cells $1^{-1}$. The Creactive protein level was raised to $165 \mathrm{mg} \mathrm{l}^{-1}$ and the albumin level was $33 \mathrm{~g} \mathrm{l}^{-1}$. The urea level was $12.2 \times 10^{6}$ mmol $\mathrm{l}^{-1}$ and the creatinine level was $448 \times 10^{6} \mu \mathrm{mol} \mathrm{l}^{-1}$. The fluid in the PD bag was pale yellow, cloudy with fibrous material and slow to drain. The PD fluid was sent for microscopy and culture, and was found to have a WCC

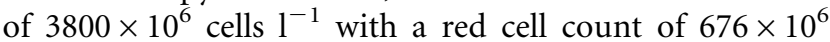
cells $1^{-1}$. WCC decreased on the three following days to

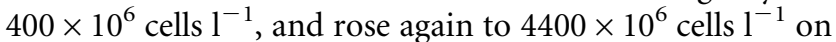
the eighth day after first examination, whereas the red cell count remained between $40 \times 10^{6}$ and $20 \times 10^{6}$ cells l $^{-1}$. No organisms were observed with the use of the Gram stain. Neosartorya was isolated from repeated peritoneal fluid and Tenckhoff catheter culture. Even after removal of the catheter a week later, before voriconazole therapy was administered, we were able to detect $N$. pseudofischeri on culture plates from peritoneal fluid, which supports the theory that the fungus cannot be a laboratory contaminant since several cultures were independently positive. Therefore, the N. pseudofischeri was considered to be the causative factor of peritonitis.

Prior to removal of the catheter on the eighth day after first examination an abdominal computed tomography (CT) scan was performed (Fig. 1), which indicated an underlying oedematous inflammation due to the fungal infection. Subsequently, after the removal of the Tenckhoff catheter, the patient was transferred to haemodialysis treatment. The therapy with $200 \mathrm{mg}$ voriconazole was administered on the fifteenth day after first examination, after the catheter had been removed, for 5 weeks twice daily after the $N$. pseudofischeri was identified.

\section{Methods}

Identification of $\boldsymbol{N}$. pseudofischeri (teleomorph of Aspergillus thermomutatus). Colony characteristics of strains were initially observed by culture on blood agar, and then by subculture on Sabouraud dextrose agar (Oxoid), and malt extract and Czapec Dox agar (Difco Laboratories) plates at $25{ }^{\circ} \mathrm{C}, 30{ }^{\circ} \mathrm{C}$ and $45^{\circ} \mathrm{C}$ for 2 to 3 days; culture at $50{ }^{\circ} \mathrm{C}$ revealed no growth. The culture on malt extract yielded abundant growth of ascomata at $25{ }^{\circ} \mathrm{C}$. Morphological features of strains were observed by lactophenol cotton blue staining. Conidiophores with mature conidia of $N$. pseudofischeri morphologically resemble A. fumigatus; however, their conidia are whitish to greyish yellow instead of a deep bluish green. Electron microscopy scanning was not available; therefore, there was no characterization of the ascospores. However, the species could not be identified by its conidia formation. To identify the species, species-specific internal transcribed spacer region (ITS1/ITS2)-based molecular identification was performed (Fujita et al., 2001; Hinrikson et al., 2005). A similarity of $100 \%$ to the N. pseudofischeri sequence with GenBank accession number EF669966.1 (ITS1/2) was determined.

Etest. An Etest (AB Biodisk) for voriconazole was used for MIC determination on RPMI agar plates (Viva Diagnostika). The Etest strips were incubated at room temperature for $20 \mathrm{~min}$ due to the storage temperature of $-20{ }^{\circ} \mathrm{C}$. The Etest was performed according to the manufacturer's instructions. Briefly, each RPMI plate was inoculated with $400 \mu \mathrm{l}$ undiluted stock inoculum (ranging from $1 \times 10^{6}$ to $5 \times 10^{6}$ c.f.u. $\mathrm{ml}^{-1}$ ) (Guinea et al., 2008). Plates were incubated at $35{ }^{\circ} \mathrm{C}$ in a humid atmosphere, and MICs were determined following incubation times of 24 and $48 \mathrm{~h}$. The MIC with the Etest was defined as the lowest voriconazole concentration at which the border of the elliptical inhibition zone intercepted the scale on the Etest strip. For Aspergillus spp. and the remaining moulds no breakpoints for the new triazoles have been established. For Candida spp., the classification of the strains for the MIC breakpoint for voriconazole was as follows: susceptible, breakpoint of $<1 \mu \mathrm{g} \mathrm{ml}^{-1}$; susceptible dose dependent, $2 \mu \mathrm{g} \mathrm{ml}^{-1}$; resistant, $>4 \mu \mathrm{g} \mathrm{ml}^{-1}$ (Pfaller et al., 2006). For our isolate of $N$. pseudofischeri the MIC for

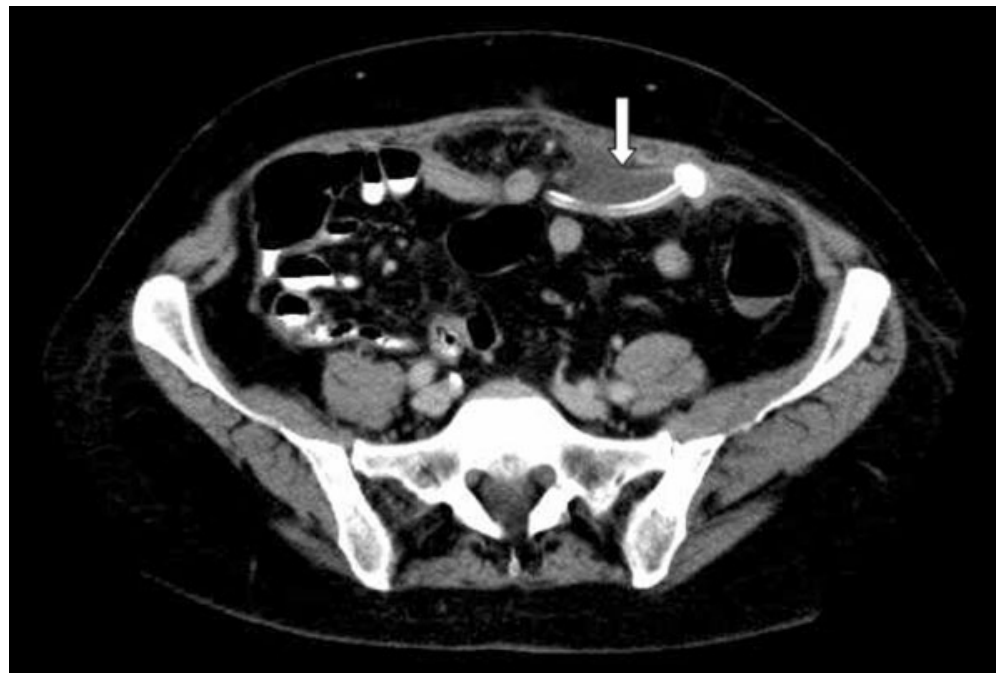

Fig. 1. Patient's abdominal CT scan on eighth day after first examination (after removal of the catheter); the white arrow indicates the oedematous inflammation of the peritoneum (peritonitis) due to N. pseudofischeri. 
voriconazole was $1.0(0.5-1.0) \mu \mathrm{g} \mathrm{m} \mathrm{m}^{-1}$. Where the elliptical inhibition zone intercepted the scale on the Etest strip between two MIC values, the MIC end point was taken as the higher value.

The determination of other antifungal MICs was carried out with the commercially available ATB fungus test (bioMérieux). The MICs obtained were $>64 \mu \mathrm{g} \mathrm{ml}^{-1}$ for flucytosine, $>128 \mu \mathrm{g} \mathrm{ml}^{-1}$ for fluconazole, $0.500 \mu \mathrm{g} \mathrm{ml}^{-1}$ for amphotericin B and $1.0 \mu \mathrm{g} \mathrm{ml}^{-1}$ for itraconazole.

\section{Discussion}

In general, FP has a prevalence of 0.1 to $10 \%$, with Candida albicans being the predominating aetiological agent (Bibashi et al., 2003; Ram et al., 2008). However, FP caused by filamentous fungi is much less common (Bibashi et al., 2003). Fungi can enter the peritoneal cavity through a catheter intraluminally or periluminally, but in a few cases a vaginal route of infection has also been observed (Stuck et al., 1986). FP is associated with a mortality rate of 5 to $25 \%$ (Bibashi et al., 1993). Risk factors for FP include recent exposure to antibiotics, immunosuppressive therapy, recent bacterial peritonitis and the presence of bowel perforation (Eisenberg et al., 1986; Ujhelyi et al., 1990).

The case we report is believed to be the first case of Neosartorya peritonitis in an elderly patient (Matsumoto et al., 2002). The clinical presentations of other reported peritonitis cases are summarized in Table 1 . A variety of Aspergillus-associated peritonitis cases have been documented (Ross et al., 1968; Arfania et al., 1981; Carpenter et al., 1982; Sridhar et al., 1990; Perez-Fontan et al., 1991;
Bibashi et al., 1993; Tanis et al., 1995; Miles \& Barth, 1995; Geiss, 1995; Park et al., 1996). Risk factors included recent antibiotic therapy, steroid therapy, recurrent peritonitis, an immunosuppressed state and hospitalization. A high mortality for FP has been reported, generally in cases where diagnosis was delayed and the catheter not removed (Tsai et al., 1991; Miles \& Barth, 1995). Over the last four decades among our 19 selected FP cases (Table 1) in 3 cases with an outcome of death the catheter was not removed, 2 patients were treated with amphotericin and the third patient had no antimycotic treatment. Only voriconazole treatment was administered in our peritonitis patient, although Balajee et al. (2005) reported that three isolates of $N$. pseudofischeri had higher MICs to voriconazole in vitro compared to $A$. fumigatus. In previous cases other antimycotics were given, preferentially amphotericin B. Another N. pseudofischeri isolate from a different case in our clinic (data not shown) also presented a low MIC $\left(<0.5 \mu \mathrm{g} \mathrm{ml}^{-1}\right)$ to voriconazole as in the case presented here.

Diagnosis can be quite difficult, since the symptoms are identical to bacterial peritonitis. A Gram stain can occasionally reveal the typical hyphae of Aspergillus. Otherwise, a positive culture result must be obtained, and preferably a positive result from repeated cultures, since Aspergillus can occur as a laboratory contaminant (Geiss, 1995). In most cases, positive cultures are obtained after 5 and 10 days. However, since delayed treatment has an increased mortality, one should not wait for the results of repeated cultures; intravenous treatment with vorico-

Table 1. A selection of reported cases of Aspergillus and Neosartorya peritonitis

\begin{tabular}{|c|c|c|c|c|c|c|}
\hline $\begin{array}{l}\text { Age of } \\
\text { patient } \\
\text { (years) }\end{array}$ & Sex & $\begin{array}{l}\text { Fungal } \\
\text { species }\end{array}$ & $\begin{array}{l}\text { Catheter } \\
\text { removal }\end{array}$ & Antimycotic & Outcome & Reference \\
\hline 22 & $\mathrm{~F}$ & A. fumigatus & No & None & Death & Ross et al. (1968) \\
\hline 61 & $\mathrm{~F}$ & A. fumigatus & No & Amphotericin B (i.v., i.p.) & Death & Arfania et al. (1981) \\
\hline 64 & $\mathrm{M}$ & A. flavus & No & Amphotericin B (i.v.) & Death & Carpenter et al. (1982) \\
\hline 40 & $\mathrm{M}$ & A. niger & Yes & Amphotericin B (i.v.) & Death & Rodriguez-Tudela et al. (1988) \\
\hline 66 & $\mathrm{M}$ & A. niger & Yes & Amphotericin B (i.v.) & CAPD & Prewitt et al. (1989) \\
\hline 49 & $\mathrm{~F}$ & A. niger & Yes & Amphotericin B (i.v.) & HD & Sridhar et al. (1990) \\
\hline 68 & $\mathrm{~F}$ & A. fumigatus & Yes & Amphotericin B (i.v.) & HD & Stein et al. (1991) \\
\hline 69 & $\mathrm{M}$ & Unidentified & Yes & Amphotericin B (i.v., i.p). + ketoconazole & CAPD & Perez-Fontan et al. (1991) \\
\hline 35 & $\mathrm{~F}$ & A. niger & Yes & Amphotericin B (i.v.) + fluconazole & CAPD & Bibashi et al. (1993) \\
\hline 68 & M & A. fumigatus & Yes & Amphotericin B (i.v.) + itraconazole & CAPD & Tanis et al. (1995) \\
\hline 61 & $\mathrm{M}$ & A. fumigatus & Yes & Amphotericin B (i.v.) + fluconazole & Death & Tsoufakis et al. (1995) \\
\hline 37 & $\mathrm{M}$ & A. niger & Yes & Amphotericin B (i.v.) + itraconazole & HD & Kitiyakara et al. (1996) \\
\hline 60 & $\mathrm{~F}$ & A. fumigatus & Yes & Ketoconazole & HD & Bren (1998) \\
\hline 64 & $\mathrm{M}$ & A. fumigatus & Yes & Amphotericin B (i.v.) & Death & Bren (1998) \\
\hline 30 & $\mathrm{~F}$ & Aspergillus sp. & Yes & Amphotericin B (i.v.) + flucytosine & Death & Bren (1998) \\
\hline 52 & $\mathrm{~F}$ & A. niger & Yes & Amphotericin B (i.v.) + fluconazole & $\mathrm{HD}$ & Basok et al. (2000) \\
\hline 8 & $\mathrm{~F}$ & N. pseudofischeri & Yes & Liposomal amphotericin B (i.v.) + itraconazole & HD & Matsumoto et al. (2002) \\
\hline 45 & $\mathrm{~F}$ & A. fumigatus & Yes & Amphotericin B (i.v.) & CAPD & Schattner et al. (2006) \\
\hline 60 & $\mathrm{~F}$ & N. pseudofischeri & Yes & Voriconazole (i.v.) & HD & This study \\
\hline
\end{tabular}

A., Aspergillus; F, female; HD, haemodialysis; i.v., intravenous; i.p., intraperitoneal; $\mathrm{M}$, male. 
nazole or amphotericin B should be administered immediately. Susceptibility testing revealed that amphotericin B is also an antifungal agent to which the organism had a low MIC of $0.5 \mu \mathrm{g} \mathrm{ml}^{-1}$. On the other hand, N. pseudofischeri had a high MIC for fluconazole and flucytosine. With respect to the removal of the catheter, there is general agreement in the literature that this is mandatory and should be performed as soon as possible (Stein et al., 1991; Perez-Fontan et al., 1991; Tanis et al., 1995; Miles \& Barth, 1995; Park et al., 1996).

In summary, we conclude that Neosartorya peritonitis is a severe form of peritonitis in CAPD patients. The need for more effective treatment and prophylaxis for fungal infections in CAPD is obvious; which means in cases where bacterial culture of peritoneal fluid is negative, this may indicate the possibility of fungal infection. If a positive fungal culture is obtained, antimycotic therapy should be started immediately, and the Tenckhoff catheter removed as quickly as possible, preferably within $24 \mathrm{~h}$ of the diagnosis, as it is mostly impossible to eradicate the infection without removing the catheter. In addition to blood agar, Sabouraud dextrose agar for Aspergillus species and Candida chromogenic agar should be used for the initial culture. A recent bacterial infection treated with antibiotics could be a predisposing factor for FP. An earlier CT scan might help raise suspicion of a fungal inflammation being present and indicate the need for administration of antimycotics as well as antibiotics. The prompt diagnosis of FP and the early institution of therapy (e.g. amphotericin B) decrease the rates of morbidity and mortality. As in our case, the removal of the catheter alone did not resolve the peritoneal inflammation within a few days (up to 1 week), which would support the administration of antimycotic therapy simultaneously to catheter removal.

\section{References}

Arfania, D., Everett, E. D., Nolph, K. D. \& Rubin, J. (1981). Uncommon causes of peritonitis in patients undergoing peritoneal dialysis. Arch Intern Med 141, 61-64.

Balajee, S. A., Gribskov, J., Brandt, M., Ito, J., Fothergill, A. \& Marr, K. A. (2005). Mistaken identity: Neosartorya pseudofischeri and its anamorph masquerading as Aspergillus fumigatus. J Clin Microbiol 43, 5996-5999.

Basok, A., Schneider, E., Hausmann, M. \& Rapoport, J. (2000). Aspergillus peritonitis in continuous ambulatory peritoneal dialysis patients. Am J Nephrol 20, 329-331.

Bibashi, E., Papagianni, A., Kelesidis, A., Antoniadou, R. \& Papadimitriou, M. (1993). Peritonitis due to Aspergillus niger in a patient on continuous ambulatory peritoneal peritoneal dialysis shortly after kidney graft rejection. Nephrol Dial Transplant 8, 185187.

Bibashi, E., Memmos, D., Kokolina, E., Tsakiris, D., Sofianou, D. \& Papadimitriou, M. (2003). Fungal peritonitis complicating peritoneal dialysis during an 11-year period: report of 46 cases. Clin Infect Dis 36, 927-931.

Bren, A. (1998). Fungal peritonitis in patients on continuous ambulatory peritoneal dialysis. Eur J Clin Microbiol Infect Dis 17, 839-843.
Carpenter, J. L., Foulks, C. J. \& Weiner, M. H. (1982). Peritoneal dialysis complicated by Aspergillus flavus peritonitis: a role for fungal antigen serodiagnosis. Nephron 32, 258-260.

Chan, T. M., Chan, C. Y., Cheng, S. W., Lo, W. K., Lo, C. Y. \& Cheng, I. K. (1994). Treatment of fungal peritonitis complicating continuous ambulatory peritoneal dialysis with oral fluconazole. Nephrol Dial Transplant 9, 539-542.

De Hoog, G. S., Guarro, J., Gené, J. \& Figueras, M. J. (2000). Atlas of Clinical Fungi, 2nd edn. Utrecht \& Reus: Centraalbureau voor Schimmelcultures \& Universitat Rovira i Virgili.

Donnelly, J. P. (2002). Symptoms and diagnosis of nosocomial fungal infections - state-of-the-art. Eur J Med Res 7, 192-199.

Eisenberg, E. S., Leviton, I. \& Soeiro, R. (1986). Fungal peritonitis in patients receiving peritoneal dialysis: experience with 11 patients and review of the literature. Rev Infect Dis 8, 309-321.

Fujita, S. I., Senda, Y., Nakaguchi, S. \& Hashimoto, T. (2001). Multiplex PCR using internal transcribed spacer 1 and 2 regions for rapid detection and identification of yeast strains. J Clin Microbiol 39, 3617-3622.

Garcia-Martos, P., Diaz, J., Castano, M., Perez, M. \& Martin, P. (1991). Peritonitis caused by Candida lusitaniae in a patient of continuous ambulatory peritoneal dialysis (CAPD). Clin Nephrol 36, 50.

Geiss, H. K. (1995). Peritoneal aspergillosis - pitfalls in the diagnosis of a rare disease. Nephrol Dial Transplant 10, 1124-1125.

Gloor, H. J., Pandolfi, S. \& Ruttimann, S. (2003). 20 years of peritoneal dialysis in a mid-sized Swiss hospital. Swiss Med Wkly 133, 619-624.

Guarro, J., Kallas, E. G., Godoy, P., Karenina, A., Gene, J., Stchigel, A. \& Colombo, A. L. (2002). Cerebral aspergillosis caused by Neosartorya hiratsukae, Brazil. Emerg Infect Dis 8, 989-991.

Guinea, J., Peláez, T., Recio, S., Torres-Narbona, M. \& Bouza, E. (2008). In vitro antifungal activities of isavuconazole (BAL4815), voriconazole, and fluconazole against 1,007 isolates of zygomycete, Candida, Aspergillus, Fusarium, and Scedosporium species. Antimicrob Agents Chemother 52, 1396-1400.

Hinrikson, H. P., Hurst, S. F., De Aguirre, L. \& Morrison, C. J. (2005). Molecular methods for the identification of Aspergillus species. Med Mycol 43 (Suppl. 1), S129-S137.

Hong, S.-B., Shin, H.-D., Hong, J., Frisvad, J. C., Nielsen, P. V., Varga, J. \& Samson, R. A. (2008). New taxa of Neosartorya and Aspergillus in Aspergillus section Fumigati. Antonie Van Leeuwenhoek 93, 87-98.

Iqbal, M. M., Sattar, H., Islam, M. N., Ahmed, A. H., Rahman, M. H., Rashid, H. U. \& Mohsi, M. (2008). Spectrum of organisms causing peritonitis in peritoneal dialysis patients - experience from Bangladesh. Adv Perit Dial 24, 40-43.

Kitiyakara, C., Sakulsaengprapha, A. \& Domrongkitchaiporn, S. (1996). The role of surgery and itraconazole in Aspergillus peritonitis in CAPD. Nephrol Dial Transplant 11, 1498.

Malloch, D. \& Cain, R. F. (1972). The Trichocomaceae: ascomycetes with Aspergillus, Paecilomyces, and Penicillium imperfect states. Can J Bot 50, 2613-2628.

Matsumoto, N., Shiraga, H., Takahashi, K., Kikuchi, K. \& Ito, K. (2002). Successful treatment of Aspergillus peritonitis in a peritoneal dialysis patient. Pediatr Nephrol 17, 243-245.

Melez, K. A., Cherry, J., Sanchez, C., Ettinger, R. B. \& Walsh, T. J. (1995). Successful outpatient treatment of Trichosporon beigelii peritonitis with oral fluconazole. Pediatr Infect Dis J 14, 1110-1113.

Miles, A. M. \& Barth, R. H. (1995). Aspergillus peritonitis: therapy, survival, and return to peritoneal dialysis. Am J Kidney Dis 26, 80-83. 
Park, S. B., Kim, K. H., Joo, I. \& Kim, H. C. (1996). Scanning electron microscopy studies of peritoneal catheter in CAPD peritonitis due to Aspergillus fumigatus. Perit Dial Int 16, 81-83.

Perez-Fontan, M., Rodriguez-Carmona, A., Fernandez-Rivera, C. \& Moncalian-Lenon, J. (1991). Aspergillus peritonitis complicating ambulatory peritoneal dialysis. Nephron 57, 493-494.

Pfaller, M. A., Diekema, D. J., Rex, J. H., Espinel-Ingroff, A., Johnson, E. M., Andes, D., Chaturvedi, V., Ghannoum, M. A., Odds, F. C. \& other authors (2006). Correlation of MIC with outcome for Candida species tested against voriconazole: analysis and proposal for interpretive breakpoints. J Clin Microbiol 44, 819-826.

Pimentel, J. D., Mahadevan, K., Woodgyer, A., Sigler, L., Gibas, C., Harris, O. C., Lupino, M. \& Athan, E. (2005). Peritonitis due to Curvularia inaequalis in an elderly patient undergoing peritoneal dialysis and a review of six cases of peritonitis associated with other Curvularia spp. J Clin Microbiol 43, 4288-4292.

Piraino, B. (1998). Peritonitis as a complication of peritoneal dialysis. J Am Soc Nephrol 9, 1956-1964.

Polo, J. R., Luño, J., Menarguez, C., Gallego, E., Robles, R. \& Hernandez, P. (1989). Peritoneal mucormycosis in a patient receiving continuous ambulatory peritoneal dialysis. Am J Kidney Dis 13, 237239.

Prewitt, K., Lockard, J., Rodgers, D. \& Harsbargen, J. (1989). Successful treatment of Aspergillus peritonitis complicating peritoneal dialysis. Am J Kidney Dis 13, 501-503.

Ram, R., Swarnalatha, G., Neela, P. \& Murty, K. V. (2008). Fungal peritonitis in patients on continuous ambulatory peritoneal dialysis: a single-centre experience in India. Nephron Clin Pract 110, 207-212.

Rodriguez-Tudela, J. L., Barros, C., Aguado, J. M., Gomez-Garcés, J. L., Velo, M. \& de Arriba, G. (1988). Aspergillus niger peritonitis. Nephrol Dial Transplant 3, 232.
Ross, D. A., Anderson, D. C., Macnaughton, M. C. \& Stewart, W. K. (1968). Fulminating disseminated aspergillosis complicating peritoneal dialysis in eclampsia. Arch Intern Med 121, 183-188.

Schattner, A., Kagan, A. \& Zimhony, O. (2006). Aspergillus peritonitis in a lupus patient on chronic peritoneal dialysis. Rheumatol Int 26, 762-764.

Sridhar, R., Thornley-Brown, D. \& Kant, K. S. (1990). Peritonitis due to Aspergillus niger: diagnostic importance of peritoneal eosinophilia. Perit Dial Int 10, 100-101.

Stein, M., Levine, J. F. \& Black, W. (1991). Successful treatment of Aspergillus peritonitis in an adult on continuous ambulatory peritoneal dialysis. Nephron 59, 145-147.

Stuck, A., Seiler, A. \& Frey, F. J. (1986). Peritonitis due to an intrauterine contraceptive device in a patient on CAPD. Perit Dial Int 6, 158.

Tanis, B. C., Verburgh, C. A., Van't Wout, J. W. \& Van der Pijl, J. W. (1995). Aspergillus peritonitis in peritoneal dialysis: case report and a review of the literature. Nephrol Dial Transplant 10, 1240-1243.

Tsai, T. J., Chen, Y. M., Hsieh, B. S., Chen, W. Y. \& Yen, T. S. (1991). Can intracatheter retention of antifungal agents cure fungal peritonitis? Two cases successfully treated without catheter removal. Perit Dial Int 11, 355-356.

Tsoufakis, G. E., latrou, C. E., Petropoulou, M., Zerbala, S. C., Vayiakou, H. A., Polychronopoulou, C. A. \& Ziroyanis, P. N. (1995). Aspergillus fumigatus peritonitis in a patient on CAPD. Perit Dial Int 15, 184-185.

Ujhelyi, M. R., Raasch, R. H., Van der Horst, C. M. \& Mattern, W. D. (1990). Treatment of peritonitis due to Curvularia and Trichosporon with amphotericin B. Rev Infect Dis 12, 621-627.

Varga, J., Vida, Z., Tóth, B., Debets, F. \& Horie, Y. (2000). Phylogenetic analysis of newly described Neosartorya species. Antonie Van Leeuwenhoek 77, 235-239. 